(2), the product of $e / m$ and $e$ should be obtained very accurately, as the errors in the values of Rydberg's constant, $c$ and $(1+m / M)$ are very small. This product corresponds to pairs of values such as

$$
\left.\begin{array}{l}
e / m \text { (e.m.u.) } 1.776 \times 10^{7} \\
e \text { (e.s.u.) } 4.867 \times 10^{-10}
\end{array}\right\} \text { or }\left\{\begin{array}{l}
1.797 \times 10^{7} \\
4.810 \times 10^{-10}
\end{array}\right.
$$

But evidence that $\mathrm{e} / \mathrm{m}$ is not appreciably larger than $1.776 \times 10^{7}$ is given by each of the following :

(i) deflection of streams of electrons;

(ii) the Zeeman effect;

(iii) $e / M$ for the hydrogen ion in electrolysis, together with $M / m$ from comparison of the Balmer and Fowler-Pickering series.

Similarly, evidence that $e$ must not be assumed appreciably larger than $4.810 \times 10^{-10}$ is given by each of the following :

(iv) the charge on an a particle;

(v) equation (1) together with h/e deduced from the photoelectric effect and from black-body radiation ; as well as

(vi) $e$ (Millikan).

This indicates that one of equations (1), (2) is in error; but the agreement of (i) and (ii) with (iii) tends to confirm equation (2), and hence throw the doubt on equation (1)

Department of Physics,

University of Reading, Aug. 19.

\section{Heat of Adsorption of Oxygen and Nitric Oxide on Gharcoal.}

MARSHALL and Bramston-Cook (J.A.C.S., 51, 2019 ; 1929) have measured the differential heat of adsorption of oxygen on activated charcoal and conclude that the heat of adsorption approaches a maximum value of 89,600 cal. per gm. mol. of oxygen as the amount of gas adsorbed approaches negligible dimensions. They refer to the work of Garner and McKie (J. Chem. Soc., 130, 2451 ; 1927 ; cf. Far. Soc., 461 ; 1926), who found at low surface concentrations a heat of adsorption of $5000 \mathrm{cal} . / \mathrm{mol}$, and observed that the heat rose to a maximum value of 71,000 cal. as the surface concentration of oxygen increased and then fell off at still higher concentrations. These values were given by an experimental arrangement in which a thermocouple was embedded in the middle of a mass

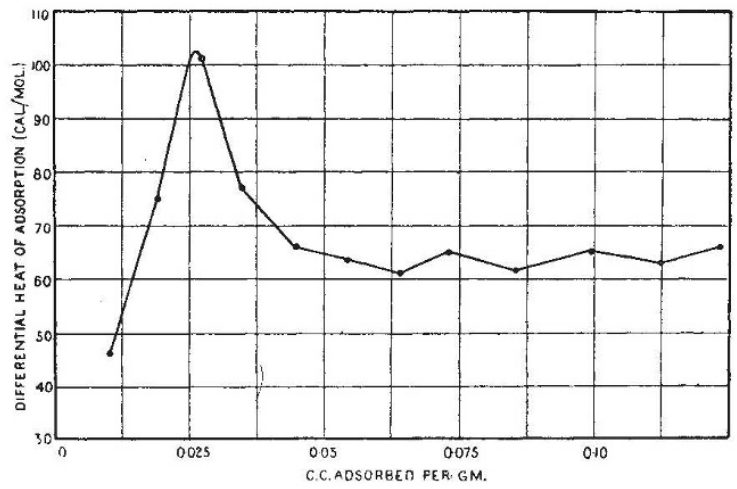

FIG. 1.-Oxygen adsorbed on charcoal at $20^{\circ} \mathrm{C}$.

of charcoal. Marshall and Bramston-Cook suggest that it is desirable to repeat this work with thermocouples placed at different points throughout the charcoal.

During the last two years, the work of McKie has been repeated, and in the course of the research measurements similar to those suggested have been made. These have brought to light the fact that at high vacua carbon is relatively a bad conductor, and have also shown that at low oxygen concentrations the gas did not distribute itself uniformly throughout the mass of the charcoal grains. On this account, the vacuum calorimeter employed by McKie was made adiabatic, thereby preventing loss of heat from the calorimeter during measurement. Under these conditions, two thermocouples, the junction of one placed in the middle and that of the other placed on the outside of the mass of charcoal, gave the same rise in temperature. With this apparatus, the curves in Figs. 1 and 2 have been obtained for the differential heats of adsorption of oxygen and nitric oxide respect. ively on charcoal.

In these experiments, $0.007-0.010$ c.c. of gas per gram of charcoal were added at a time. For oxygen

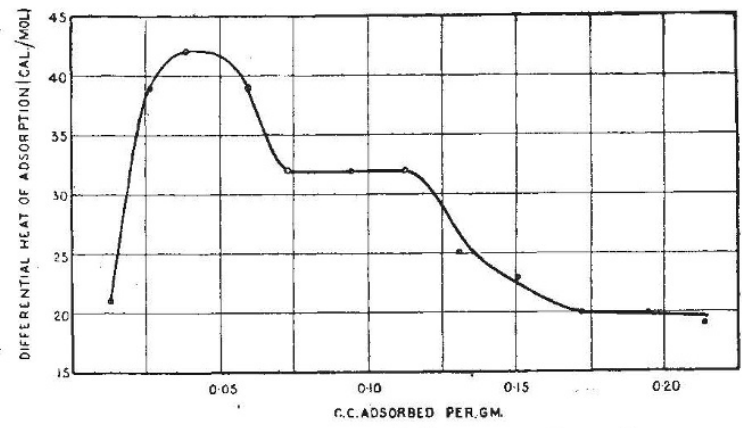

FIG. 2.-Nitric oxide adsorbed on charcoal at $20^{\circ} \mathrm{C}$.

adsorbed on charcoal, the heat rises from $46 \mathrm{cal} . / \mathrm{mol}$. to $100 \mathrm{cal} . / \mathrm{mol}$. by the third admission and then falls to 65 cal., at which value it remains constant except for a curious type of alternation. The maximum occurs when 0.025 c.c. of oxygen per gram of charcoal had been adsorbed. This maximum shifts after long. continued heat treatment at $950^{\circ} \mathrm{C}$. to lower surface concentrations. The American authors in their measurements added 0.03 c.c. oxygen per gram charcoal at a time, so that their surface concentrations were greater than ours. Also they gave the charcoal a preliminary treatment at $1000^{\circ} \mathrm{C}$. for 60 hours.

For the same charcoal, the maximum heat of adsorption of nitric oxide occurs at somewhat higher surface concentrations than for oxygen. The alternation beyond the maximum does not occur for nitric oxide. There is a definite step on the curve indicating the presence of areas of different activities on the charcoal surface.

Kistiakowski and Taylor (J.A.C.S., 49, $2200 ; 1927$ ) have observed similar maxima on the curves for the differential heats of adsorption of hydrogen on metals. We have not been able to observe the effect in the reaction of oxygen with finely divided copper, but this may be due to the fact that the surface concentrations employed were too high. With oxygen on copper, the maximum heat occurs at the lowest surface concentrations used.

The maximum value for the heat of adsorption of oxygen on charcoal which was obtained for single admissions of gas occurs when 0.12 c.c. oxygen is added per gram of charcoal. The value, 89 cal., agrees with that of Marshall and Bramston-Cook. The value obtained probably depends on the nature of the charcoal.

It is difficult to decide whether the above phenomena are due to activation of the carbon surface or to incompleteness of the reaction between oxygen and carbon at low surface concentrations. The experimental evidence is, however, in favour of the latter hypothesis.

H. I. BuLl.

University, Bristol, July 30. 